\title{
Modelling the Full X-ray Spectra of MCG-6-30-15 with a Relativistic Reflection Model
}

\section{Chia-Ying Chiang*}

Institute of Astronomy, University of Cambridge - Madingley Road, Cambridge CB3 OHA

E-mail: cychiangeast.cam.ac.uk

\section{Andrew C. Fabian}

Institute of Astronomy, University of Cambridge - Madingley Road, Cambridge CB3 OHA

E-mail: acflast.cam.ac.uk

\begin{abstract}
The AGN MCG-6-30-15, which shares many similarities with narrow line Seyfert 1 (NLS1) galaxies, shows a broad Fe K- $\alpha$ line that is generally explained as reflection from the inner part of an accretion disc around a fast spinning black hole. The flux of the line was expected to follow that of the powerlaw component, but little variability was found. The disconnection of continuum and line variability may be caused by strong gravitational light bending. However, some workers have built an absorption model and attribute most variability to the warm absorbers. They claim relativistic reflection is not needed to explain the source behaviour and the hard excess. We reexamine the XMM-Newton, Chandra and Suzaku data taken in different epochs and construct a model consisting of several warm absorbers, together with a relativistic reflection component that explains both the soft and hard excesses. Our model works well on all data, implying it provides a consistent interpretation of the broadband spectrum of MCG-6-30-15.
\end{abstract}

Narrow-Line Seyfert 1 Galaxies and Their Place in the Universe

Milan, Italy

April 04-06, 2011

\footnotetext{
${ }^{*}$ Speaker.
} 


\section{Introduction}

A broad iron line was first discovered by the Advanced Satellite for Cosmology and Astrophysics (ASCA) in the X-ray spectrum of the Seyfert I galaxy MCG-6-30-15 [11]. Since then MCG-6-30-15 has become one of the most studied AGNs. The soft X-ray spectrum of MCG-630-15 has complex structures usually attributed to warm absorbers [9]. [4] presented evidence of dusty warm absorbers in the Chandra High Energy Transmission Grating Spectrometer (HETGS) observation. The $0.7 \mathrm{keV}$ drop can be interpreted as a combination of resonance absorption lines redward of the O VII edge and an Fe I L-shell absorption edge, caused by dust grain iron oxides or silicates. The presence of dust is in agreement with the optical observations of [10]. The total Xray spectrum of MCG-6-30-15 could hence be illustrated by a powerlaw continuum, the reflected component from the cool disc, and absorptions by warm absorbers.

An initially puzzling lack of line variations during rapid continuum variations $[1,5]$ aroused interest. [8] showed that if the illuminating source moves within the innermost area around the black hole where there are strong general relativistic light-bending effects, then the flux of the continuum varies but the iron line could stay unchanged. This gravitational light-bending model interprets the lack of variability of the iron line, despite the observed variability of the continuum, as due to changes in the location of the powerlaw component. [3], on the other hand, suggested a different view with an absorption-dominated model which could explain the X-ray spectra of MCG6-30-15 as well. The warm absorbers might imitate the "red wing" structure seen in the iron line energy band, and variations of continuum are hence due to warm absorbers but not of the powerlaw component. [6,7] further extended the absorption model to build a complex " $3+2$ " model which consists of five absorbing zones, two of which are 'partial-covering'. They also claimed that the strong emission found at high energies (above 10keV), known as the "hard excess", cannot be explained with a simple disc reflection scenario and concluded that high-column partial-covering absorption dominates the hard excess in MCG-6-30-15. With 3 percent systematic errors included, they claim that this model reproduces the variability and $X M M$ spectra of MCG-6-30-15. The partial covering component responsible for the red wing shape produces no other observed features at $\mathrm{CCD}$ resolution.

We start by investigating the difference spectra of the three datasets and then study the full real spectra. The grating data of Chandra, the energy resolution of which is better than other instruments, were used to study the effects of warm absorbers and determine their shape. A reflection model with absorbing zones was applied to these observations to test the robustness of the model. Changes of ionization state in at least one absorbing zone are required to fit data taken at different epochs. The partial-covering absorption model might offer an alternative explanation for the variability and spectrum of MCG-6-30-15, but the reflection model, together with the light-bending model, is fully consistent with the data and provides a more physical point of view.

\section{Difference Spectra}

We create a new spectrum by subtracting a low-flux spectrum from a high-flux spectrum. The resulting spectrum, which is called a difference spectrum, offers information on the nature of the variability because constant components in the original spectrum are removed. Figure 1 shows the 


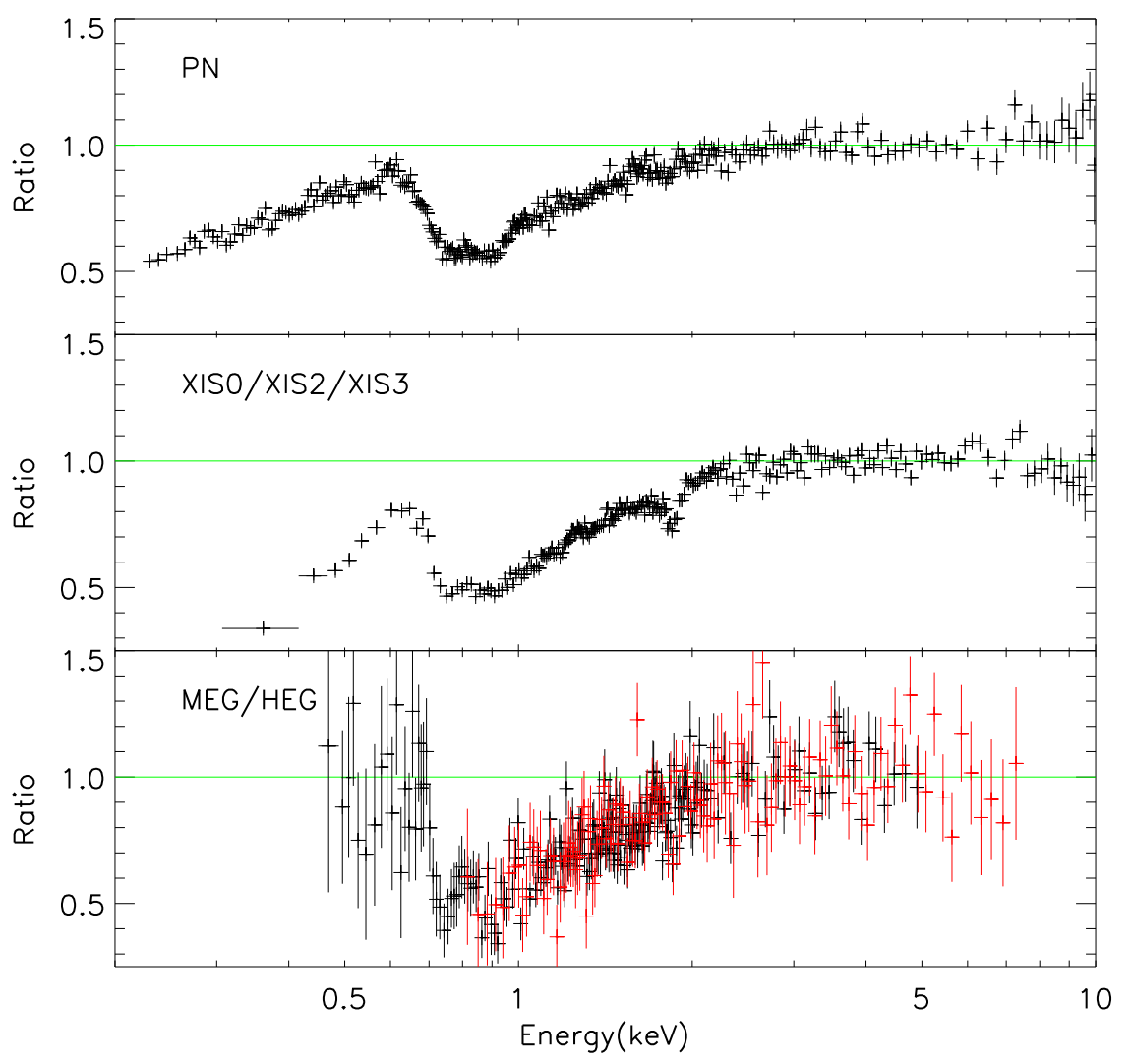

Figure 1: The figure shows the difference spectra of the three satellites plotted as energy against the data/model ratio. The model of a power-law with Galactic absorption modification fitted across 3-10 keV (3-5 keV for Chandra MEG). The difference spectra from top to bottom belong to XMM-Newton PN camera, Suzaku FI XIS detectors and Chandra HETGS, respectively. The absorption structure around $1.8 \mathrm{keV}$ in the middle panel is possibly caused by the detector. These difference spectra show a highly similar structure between $0.5 \mathrm{keV}$ and $5 \mathrm{keV}$.

difference spectra of XMM-Newton PN camera, Suzaku XIS detectors, and Chandra HETGS (HEG data in red) as a ratio to a powerlaw modified by Galactic absorption $\left(N_{\mathrm{H}}=4.06 \times 10^{20} \mathrm{~cm}^{-2}\right)$ fitted across the 3-10 keV range. The structure of the spectra from all satellites is highly similar to each other. The energy band above $3 \mathrm{keV}$ can be simply fitted by a powerlaw with Galactic absorption, which means the warm absorbers have little effect on the spectrum above $3 \mathrm{keV}$, particularly in the $\mathrm{Fe} \mathrm{K}$ band, in contrast with the band below $3 \mathrm{keV}$.

\section{Warm Absorbers}

The spectrum of MCG-6-30-15 shows complicated absorption in the soft X-ray band. Previous studies confirmed a highly ionized $(\log >3.5)$ fast component $\left(\sim-1900 \mathrm{~km} \mathrm{~s}^{-1}\right)$, and two slow components $\left(\sim-100 \mathrm{~km} \mathrm{~s}^{-1}\right)$ with different ionization states. [2] reported a fourth "local" $(\mathrm{z}=0)$ absorbing zone. We generated the ionized absorber models using XSTAR. The source luminosity 

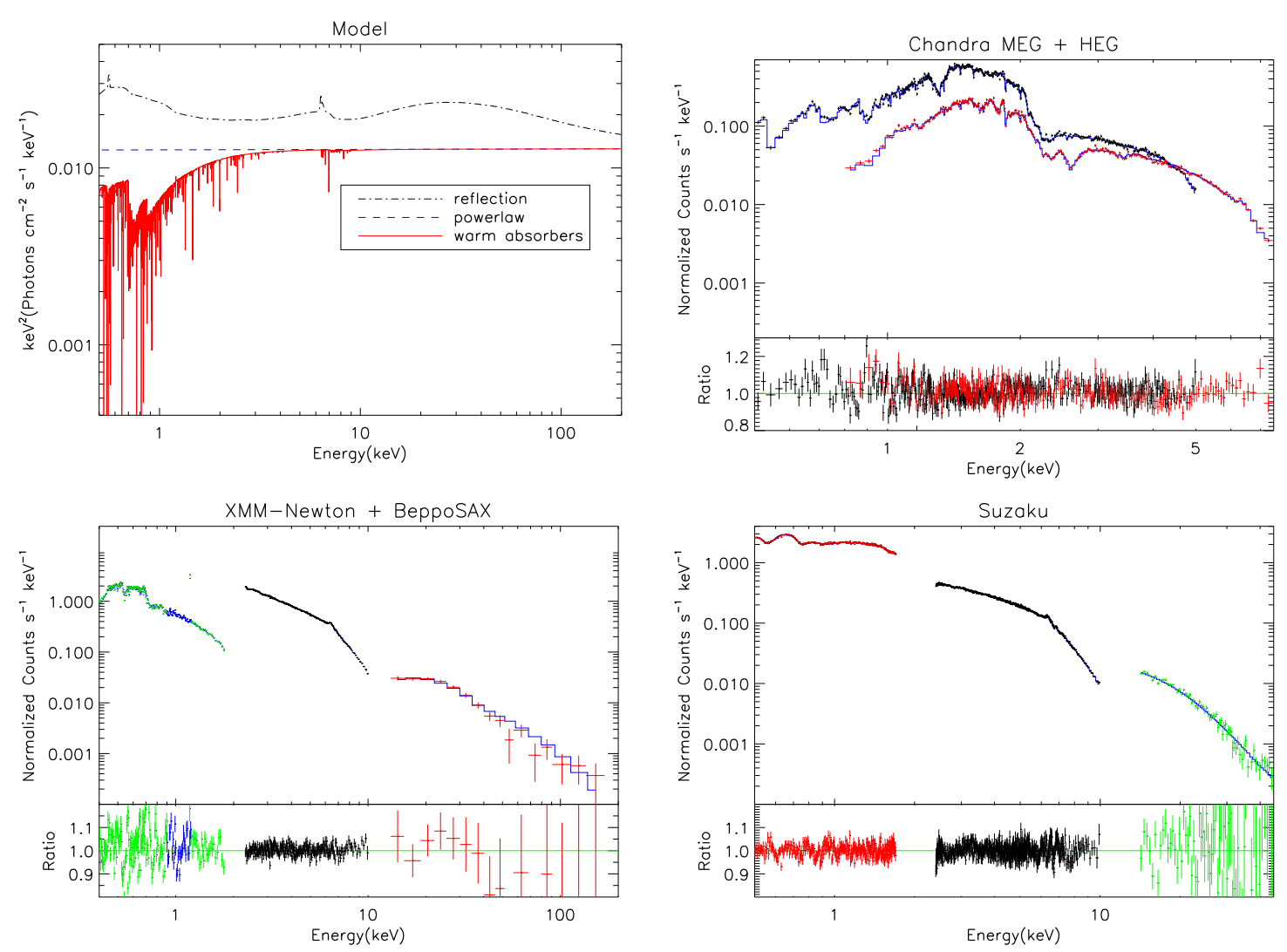

Figure 2: The upper-left figure shows the decomposed components of the 4-absorption model. The black dot-dash line above shows the reflection component consisted of a blurred reflection, a neutral reflection and a narrow Gaussian line. The blue dash line represents a $\Gamma=2.0$ powerlaw and the red solid line shows the effects of the warm absorbers. Note that the reflection component has been moved above for clarity. The rest three figures are the results fitted by the reflection model of data from different satellites. The upper panels show data points (black, red and green dots) and the best-fitted model (blue lines). The lower panel shows the data/model ratio of this fitting, and the data points have been re-binned for clarity. The model is in excellent agreement with the Chandra data above $1 \mathrm{keV}$ and struggles slightly below $1 \mathrm{keV}$, but main features are fitted. It could be clearly seen that the model fits the high energy data well in both lower figures.

between 1 and 1000 Ryd was assumed to be $2 \times 10^{43} \mathrm{erg} \mathrm{s}^{-1}$ [12] with a powerlaw spectrum shape, the gas density was set to be $10^{12} \mathrm{~cm}^{-3}$, and the covering factor equal to 1 .

\section{Model Fitting}

A relativistic blurred reflection component, a neutral reflection component, another narrow line modelled by ZGAUSS, and the fast ionization zone which produces Fe XXV and Fe XXVI absorption lines are needed to fit the complicated broad iron line profile in MCG-6-30-15. The three small figures above show the iron line profiles fitted by the best-fitted model. We fit the low energy spectra with the reflection model constructed above and the XSTAR grids we created. [4] presented evidence of dusty warm absorbers and interpreted the $0.7 \mathrm{keV}$ drop as a combination of 

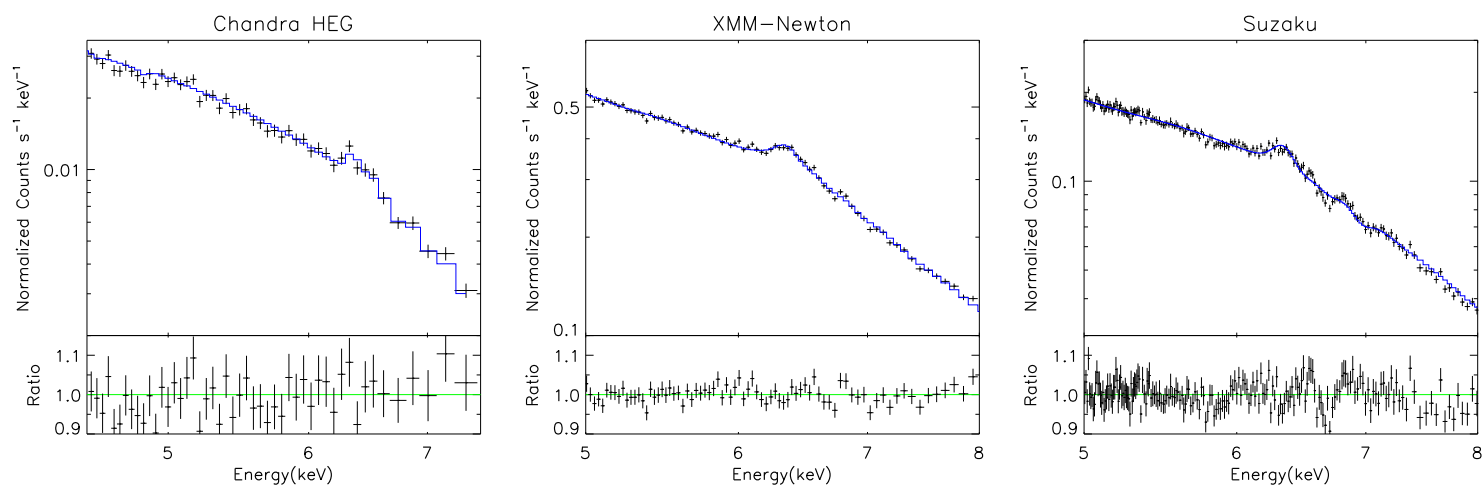

Figure 3: These figures show the zoom-in iron line fitted by the best-fitting model. Line profiles from left to right belong to Chandra HEG , XMM PN, and Suzaku respectively.

absorption lines and an Fe L-shell edge. We model the Fe L edge using dust in TBnew. Figure 2 shows the components of the best-fitting model and the results of different datasets.

\section{Conclusion}

The "local" ionization zone is not need in the Suzaku spectrum since it does not have the sharp energy resolution as Chandra. The reflection model can interpret the spectra of MCG-6-30-15 in the range from $0.5-200 \mathrm{keV}$ without any partial-covering absorbers. We consider this to be a more consistent physical scenario, since the energy release in both models occurs very close to the black hole, where relativistic reflection is expected.

\section{References}

[1] Fabian A. C., Vaughan S., Nandra K., Iwasawa K., Ballantyne D. R., Lee J. C., De Rosa A., Turner A., Young A. J., 2002, MNRAS, 335, L1

[2] Holczer T., Behar E., Arav N., 2010, ApJ, 708, 981

[3] Inoue H., Matsumoto C., 2003, PASJ, 55, 625

[4] Lee J. C., Ogle P. M., Canizares C. R., Marshall H. L., Schulz N. S., Morales R., Fabian A. C., Iwasawa K., 2001, ApJL, 554, L13

[5] Matsumoto C., Inoue H., Fabian A. C., Iwasawa K., 2003, PASJ, 55, 615

[6] Miller L., Turner T. J., Reeves J. N., 2008, $A$ \& A, 483, 437

[7] Miller L., Turner T. J., Reeves J. N., 2009, MNRAS, 399, L69

[8] Miniutti G., Fabian A. C., 2004, MNRAS, 349, 1435

[9] Otani C., Kii T., Reynolds C. S., Fabian A. C., Iwasawa K., Hayashida K., Inoue H., Kunieda H., Makino F., Matsuoka M., Tanaka Y., 1996, PASJ, 48, 211

[10] Reynolds C. S., Ward M. J., Fabian A. C., Celotti A., 1997, MNRAS, 291, 403

[11] Tanaka Y., Nandra K., Fabian A. C., Inoue H., Otani C., Dotani T., Hayashida K., Iwasawa K., Kii T., Kunieda H., Makino F., Matsuoka M., 1995, Nature, 375, 659 
[12] Young A. J., Lee J. C., Fabian A. C., Reynolds C. S., Gibson R. R., Canizares C. R., 2005, ApJ, 631, 733 